\title{
Les langues entre elles dans la Jérusalem ottomane (1880-1914). Les écoles missionnaires françaises
}

Karène Sanchez-Summerer

\section{CpenEdition}

Journals

Édition électronique

URL : https://journals.openedition.org/dhfles/864

DOI : $10.4000 /$ dhfles.864

ISSN : 2221-4038

Éditeur

Société Internationale pour l'Histoire du Français Langue Étrangère ou Seconde

Édition imprimée

Date de publication : 1 décembre 2009

Pagination : 119-143

ISSN : 0992-7654

Référence électronique

Karène Sanchez-Summerer, «Les langues entre elles dans la Jérusalem ottomane (1880-1914). Les écoles missionnaires françaises ", Documents pour l'histoire du français langue étrangère ou seconde [En ligne], 43 | 2009, mis en ligne le 16 janvier 2011, consulté le 27 mai 2021. URL : http:// journals.openedition.org/dhfles/864 ; DOI : https://doi.org/10.4000/dhfles.864

Ce document a été généré automatiquement le 27 mai 2021.

(C) SIHFLES 


\title{
Les langues entre elles dans la Jérusalem ottomane (1880-1914). Les écoles missionnaires françaises
}

\author{
Karène Sanchez-Summerer
}

1 Si l'on considère l'ensemble des facteurs linguistiques du "marché langagier » à Jérusalem de 1880 à 1914 (la compétence, la fréquence d'utilisation familiale, relationnelle, professionnelle, culturelle, éducative, ainsi que la perception du français notamment, $c f$. Laponce $1006: 12$ ), on peut évoquer une situation favorable à la langue française. Vers la fin de la période, l'un de ses principaux représentants, le prieur de l'école biblique et archéologique, déclare en effet, «A Jérusalem, le voyageur pouvait descendre dans n'importe quel hôtel, pénétrer dans les magasins les plus disparates, il était sûr d'être compris. Le français était la langue officieuse que chacun devait posséder pour entrer dans la société et se mettre en contact avec les étrangers ${ }^{1} »$ (Dhorme 1922 : 0172).

2 L'identité linguistique est alors plutôt plurielle à Jérusalem (Khalidi 1997: 87)². Le système éducatif lui-même est divisé (et le restera), diversité qui favorise dans une certaine mesure les débats sur les rapports à la langue maternelle (et à la culture), autour du rapport à la France, et à l'Occident de manière plus générale, débats qui peuvent parfois tourner à la polémique ${ }^{3}$. A l'époque ottomane, les minorités jouissent d'une liberté linguistique et religieuse, même si la tolérance religieuse elle-même est réglée sur les préceptes de l'islam.

Le sort de la ville est inévitablement lié à celui de la Palestine, aux directives linguistiques qui sont élaborées à Constantinople; le pouvoir ottoman est favorable au français dont il adopte le modèle pour ses élites un peu partout au Levant; Jérusalem ne fait guère exception ${ }^{4}$. Dans le dernier quart du XIX ${ }^{e}$ siècle, la ville est réputée pour le multilinguisme de ses écoles. Le nombre de communautés religieuses présentes dans la ville sainte ne fait que renforcer ce plurilinguisme, "l'inflation scolaire» ayant engendré une forme "d'inflation linguistique», la langue étant un terrain d'affrontement supplémentaire entre acteurs européens ${ }^{5}$. Dès le milieu du XIX ${ }^{e}$ siècle, 
et plus particulièrement sous la période des Tanzimat (réformes), Jérusalem devient la capitale de l'ensemble de la Palestine, en plus du statut de ville sainte qu'elle a déjà acquis ${ }^{6}$; les différentes puissances européennes, profitant de ce nouveau statut de la ville, subventionnent l'installation de plusieurs ordres religieux destinés à promouvoir l'éducation et l'assistance, "missionnaires de la langue ${ }^{7}$ ». Le plurilinguisme urbain palestinien s'oppose au monolinguisme rural.

Avant la guerre de 1914-18, la population scolaire de Palestine sur laquelle s'exerce l'influence des Frères est relativement importante (10 000 élèves dans 8 écoles), celle des Sœurs est tout aussi importante proportionnellement au taux de filles scolarisées ${ }^{8}$, à un moment où émerge une politique culturelle française qui aboutit à une « francophonisation » (Laurens $2004: 141)^{9}$.

Dans un tel contexte, les langues entretiennent des relations de confrontation, domination, répulsion/ attraction, la même langue pouvant avoir différentes connotations. "Les échanges linguistiques sont aussi des rapports de pouvoir symboliques de communication [...] où s'actualisent les rapports de force entre les locuteurs ou leurs groupes respectifs » (Bourdieu 1982 : 14). Ainsi nous tenterons de voir quels sont les contacts entre les langues, les représentations attachées au français, sa place dans les curricula par rapport aux autres langues, la langue et son apprentissage étant un « instrument d'action et de pouvoir » (ibid.).

\section{La mosaïque multilingue hiérosolymitaine}

\section{Diversité des élèves et des enseignants}

6 Le français, qualifiée de "langue de cœur » selon le P. Dhorme, devient une langue de communication assez importante ${ }^{10}$, surtout après la réouverture du Patriarcat latin à Jérusalem en 1847 (l'année d'après voit déjà l'établissement des Sœurs de Saint Joseph), qui donne l'élan à de nombreuses missions catholiques françaises ${ }^{11}$. Pour les autorités françaises, la place particulière du français tient au rôle protecteur de la France, rôle séculaire qui est synonyme pour ses représentants d'une supériorité de sa mission culturelle. "Nos intérêts en Palestine ne sont pas de même sorte que les intérêts de quelque autre nation [...] nous étudierons avec profit les divers organes étrangers qui nous font concurrence, mais ce ne sera point pour les imiter servilement " (Pernot 1912 : 124). Durant la période ottomane, plusieurs études sont lancées pour convenir des causes de la grandeur de la France, elles mettent en avant l'argument de l'influence de l'antériorité de la présence française comme justificatif de la présence et du rôle de la langue française à préserver et à diffuser ${ }^{12}$. La langue française apparaît comme la langue semi-officielle, elle est présente parmi différentes catégories de la population, surtout parmi la catégorie de la population la plus aisée ${ }^{13}$. La ville connaît une augmentation du nombre de ses habitants ${ }^{14}$; dans ce contexte, les élites urbaines veulent donner à leurs enfants une éducation "à l'européenne ». On remarque en effet que très rapidement le fils du gouverneur ottoman est inscrit au collège, à une période où les élèves musulmans sont minoritaires chez les Frères ${ }^{15}$. Le développement du tourisme et des pèlerinages favorise l'apprentissage de plusieurs langues étrangères qui garantit une insertion des futurs élèves dans le tissu économique, il constitue une des clés de la promotion sociale. Avec la création des écoles privées, on passe progressivement d'une éducation réservée aux élites, et de nature plutôt religieuse à 
une éducation à l'européenne, où l'apprentissage des langues étrangères est prédominant, bien que le taux d'analphabétisme demeure important ${ }^{16}$. Cet apprentissage assure en effet « la clef d'un avenir riche et commode [...] aux pèlerins vient se joindre chaque année avec une sûreté régulière le flot montant des touristes de tous les pays $\|^{17}$. L'accession à la modernité exige la maitrise d'une langue européenne (Laurens 2004: 107). "Devenir drogman, c'est-à-dire [...] apprendre des langues étrangères, le français d'abord, puis l'anglais, est aujourd'hui dans la tête de beaucoup de garçons $»^{18}$.

7 La diversité linguistique n'est pas synonyme, pendant l'époque ottomane, de handicap. Cette diversité vient entre autres de l'appartenance religieuse et ethnique à différents groupes. Les élèves scolarisés dans les établissements missionnaires français sont issus essentiellement des communautés latines mais aussi de la communauté arménienne (catholique et orthodoxe), l'arabe, mais aussi le grec et l'arménien sont des langues parlées dans les foyers des élèves. Pour la plupart des chrétiens, le français est « une langue refuge » associée au Protectorat français. Pour les musulmans, le français est indispensable pour faire carrière à l'intérieur du système ottoman rénové (c'est la deuxième langue d'administration de l'empire). Ainsi, ces établissements (surtout leurs sections payantes) s'ouvrent progressivement aux élèves musulmans et juifs ${ }^{19}$. Dès le départ, de nombreux orthodoxes de toutes les obédiences s'inscrivent dans cet établissement catholique dont la réputation dépasse rapidement les limites urbaines de Jérusalem. Les élèves sont donc parfois en situation de diglossie. Les établissements missionnaires pour garçons sont ouverts à toutes les communautés, et sont perçus comme moins prosélytes que les protestants.

On pourrait évoquer pour Jérusalem, une inflation scolaire $»^{20}$, les religieux européens soutenant ce dispositif scolaire. Les religieux français sont de loin les plus importants en nombre et davantage tournés vers le secteur éducatif que les Italiens.

Tab. 1 : Nombre de religieux à Jérusalem en 1895 (d'après V. Cuinet)

\begin{tabular}{r|rr|rr|rr|rr|rr}
\hline \hline & \multicolumn{2}{|c}{ Français } & \multicolumn{2}{c}{ Italiens } & \multicolumn{2}{c}{ Allemands } & \multicolumn{2}{c}{ Grecs Unis } & \multicolumn{2}{c}{ Arabes } \\
& couv. & relig. & couv. & relig. & couv. & relig. & couv. & relig. & couv. & relig. \\
\hline hommes & 5 & 72 & 3 & 118 & 1 & 2 & 1 & 3 & & \\
femmes & 8 & 123 & 1 & 6 & 1 & 4 & & & 1 & 10 \\
\hline total & 13 & 195 & $\mathbf{4}$ & $\mathbf{1 2 4}$ & $\mathbf{2}$ & $\mathbf{6}$ & $\mathbf{1}$ & $\mathbf{3}$ & $\mathbf{1}$ & $\mathbf{1 0}$ \\
\hline \hline
\end{tabular}

Dans ce paysage de concurrence éducative, les établissements français font figure de pionniers, les Frères sont perçus comme des pionniers de l'éducation pour garçons, pour les filles, il s'agit des Sœurs de Saint Joseph, arrivées dès 1848. Les ordres français assurent en effet la majorité de l'enseignement aux filles (93\% de l'enseignement catholique en 1895 et $73 \%$ de l'enseignement privé- catholique, orthodoxe et protestant). La femme palestinienne est perçue comme la gardienne du foyer et de ses valeurs (glorification de la sphère domestique et importance de la maternité), mais avec la naissance des classes moyennes urbaines, comme en Europe, nait la revendication d'éducation et d'émancipation(Cuinet 1895) ${ }^{21}$.

Les établissements missionnaires sont soucieux dès le début du XXe siècle de s'adapter aux nouvelles implantations géographiques des élites et des classes moyennes, élargir les inscriptions aux classes moyennes environnantes et aux communautés musulmanes et juives et proposer un apprentissage des langues souhaitées par les parents ${ }^{22}$. Les 
écoles étrangères sont nombreuses, plus nombreuses pour les garçons que pour les filles ${ }^{23}$.

11 La concurrence avec les établissements anglais apparaît très tôt, par le biais des missions protestantes. ${ }^{24}$ Les initiatives allemandes (couronnées par le voyage de l'empereur Guillaume II à Jérusalem en 1898), italiennes et russes sont importantes jusqu'en 1918; la littérature catholique française de l'époque fait référence aux « invasions » des missions chrétiennes concurrentes (Prudhomme $2006: 37$ ). C'est en 1858 qu'ouvre le consulat de Russie et en 1859 le Comité de Palestine. Il s'agit pour ces deux organismes de soutenir, créer et protéger l'orthodoxie au Proche-Orient. Ce mouvement est accompagné par la création d'une presse savante de vulgarisation et de propagande, de la création d'écoles et d'hôpitaux. Quatre ans après l'ouverture du collège des Frères, c'est l'ouverture solennelle de la Société russe (Astafieva 2003 : 45).

Jérusalem est sans doute perçue pendant cette période ottomane par plusieurs voyageurs européens comme "une Babel linguistique sur fond d'arabe et de français dominant» (Nicault 1999: 103). Les élèves scolarisés dans ces établissements missionnaires jouent avec ces différentes cartes linguistiques.

\section{Langues véhiculaire et vernaculaire dans le système éducatif}

Tab. 2 : Etablissements pour garçons et langues d'apprentissage (ACJ, d'après les données de I'Institut archéologique allemand, 1905)

\begin{tabular}{|c|c|c|c|}
\hline Ecoles & $\begin{array}{l}\text { Année de la } \\
\text { fondation } \\
\text { modèle éduc. }\end{array}$ & Langues & $\begin{array}{c}\text { heures } \\
\text { par } \\
\text { semaine }\end{array}$ \\
\hline $\begin{array}{l}\text { Turques } \\
\text { idadi (inf.) }\end{array}$ & & arabe & $6 \mathrm{~h}$ arabe \\
\hline rushdiye (m) & 1902 & & $6 \mathrm{~h}$ puis $2-3 \mathrm{~h}$ arabe \\
\hline ibtindaie (élé.) & 1890 (Eur.) & français, turc, persan & \\
\hline Orthodoxes & & & \\
\hline $\begin{array}{l}\text { collège (inf.) } \\
\text { collège (sup.) }\end{array}$ & $\begin{array}{c}1900 \\
1903 \text { reorg. }\end{array}$ & $\frac{\text { grec }}{\text { grec, arabe }}$ & $\begin{array}{c}\text { 1è a: } 7 \mathrm{~h} \text { grec, arabe } \\
\text { à partir de } 2 \text { è a : } 10 \mathrm{~h} \text { arabe, grec } \\
\text { 3è a : } 3 \mathrm{~h} \text { français }\end{array}$ \\
\hline $\begin{array}{l}\text { Catholiques lat. } \\
\text { Franciscains } \\
\text { schola paroc. }\end{array}$ & $\begin{array}{c}1889 \\
\text { (réouv.) }\end{array}$ & arabe & $\begin{array}{c}\text { 1è a: } 2 \mathrm{~h} \text { arabe, français, anglais } \\
\text { 3è a : Ih italien }\end{array}$ \\
\hline $\begin{array}{r}\text { FEC } \\
\text { école } \\
\text { collège }\end{array}$ & $\begin{array}{l}1878 \\
1904\end{array}$ & français & $\begin{array}{c}12 \text { à } 16 \mathrm{~h} \text { français } \\
5 \mathrm{~h} \text { anglais, } 12 \mathrm{~h} \text { arabe } \\
12 \text { à } 16 \mathrm{~h} \text { français }\end{array}$ \\
\hline $\begin{array}{l}\text { Protestantes } \\
\text { CMS école } \\
\text { collège }\end{array}$ & & $\begin{array}{c}\text { arabe } \\
\text { anglais }\end{array}$ & $\begin{array}{c}9 \mathrm{~h} \text { anglais, } 6 \mathrm{~h} \text { arabe } \\
\text { 3h français }\end{array}$ \\
\hline $\begin{array}{r}\text { St. George } \\
\text { Boys Day } \\
\text { School }\end{array}$ & 1899 & anglais & $\begin{array}{l}3,3 \mathrm{~h} \text { arabe } \\
2,7 \mathrm{~h} \text { français }\end{array}$ \\
\hline $\begin{array}{c}\text { école allem. } \\
\text { collège allem. }\end{array}$ & 1902 & allemand, arabe & $6 \mathrm{~h}$ allemand \\
\hline
\end{tabular}

Si Jérusalem connaît un plurilinguisme à langues dominantes minoritaires ${ }^{25}$, dans le système scolaire, ce sont certaines langues européennes qui dominent ${ }^{26}$. Vivant dans une partie reculée de l'empire ottoman, les Palestiniens pratiquent peu le turc, même s'il est obligatoire, la plupart des écoles missionnaires ne proposent que quelques heures par semaine (Frijhoff 2008: 425). Il s'agit essentiellement de systèmes 
d'éducation nationaux européens, le système gouvernemental est peu développée ${ }^{27}$. La période ottomane apparaît comme un « âge d'or » du français (même si cela représente peu de locuteurs en chiffres absolus). Au sein des différentes écoles, le français paraît en effet exercer une pression éliminatoire sur les autres langues européenne. Le français et l'arabe apparaissent comme des «normes linguistiques» (Bourdieu 1982: 27) dans les différents apprentissages. Celui du français s'effectue en contexte de tensions linguistiques croissantes, de "guerre des langues" hiérosolymitaine ${ }^{28}$. Le français jusqu'à la fin du XIXe apparaît comme une langue de Koinè. Après 1880, peu continuent à Istanbul pour être éduqués. Les langues apprises sont peu pratiquées en dehors de certaines activités professionnelles (pour le français, poste, banque, compagnie d'électricité). Cet enseignement n'est pas assorti, contrairement à la situation libanaise, d'un apprentissage auprès de précepteurs francophones.

Le modèle éducatif français s'oppose en partie au modèle allemand (et anglais), l'argumentaire fondamental étant relatif à l'image et à l'importance de la langue. Ce dernier estime en effet que le multilinguisme éloigne de l'efficacité économique et qu'elle ne bénéficie qu'aux pays européens. Selon ce modèle éducatif, la langue maternelle de l'élève doit être parfaitement maitrisée, avant toute autre langue, « dans la langue maternelle sont les racines de la force ${ }^{29}$. Il reconsidère la position des éducateurs vis à vis de leur langue nationale et de la langue maternelle des élèves. Il agite l'épouvantail de la « confusion linguistique » voire de la déperdition linguistique, comme un signe de dégénérescence de la société palestinienne et estime que les manuels d'histoire, de géographie, de leçons de choses devraient avoir «une forme originale et une couleur locale». Le but des écoles russes est de donner des connaissances de base sur l'orthodoxie, enseigner le russe (la langue est un moyen de russification) mais aussi la langue arabe, considérée comme indispensable puisque permettant aux élèves de participer aux services religieux et à la vie sociale (Astafieva $2003: 65)$.

cadres de la société palestinienne ottomane réclament un enseignement du français plus pratique, plus professionnel. Progressivement, depuis la fin des années 1890, le collège acquiert une réputation pour la formation professionnelle de ses élèves. Des institutions intègrent le français comme langue de sélection professionnelle, il s'agit par exemple des succursales de la banque impériale ottomane (de capital occidental) et du Crédit Lyonnais ${ }^{30}$.

Pourtant dès l'époque ottomane, le problème de la formation supérieure est mentionné. Son absence est rapidement perçue comme un handicap au niveau territorial pour les débouchés en Palestine même, notamment en langue française (Pernot 1912 : 126). Le collège anglais du Bishop Gobat passe rapidement des accords avec le Syrian Protestant College (qui deviendra l'université américaine de Beyrouth), fondé en 1866 et assure ainsi une postérité au niveau de l'enseignement supérieur à l'enseignement anglophone ${ }^{31}$. 


\section{Le français en concurrence, langue nationale et catholique?}

\section{Des établissements vecteurs et fers de lance de la France}

17 La Palestine est considérée comme « la plus française des terres d'Orient » et le reste même après les lois de 1901, 1904 et 1905. Le développement des congrégations enseignant le français est rapide ${ }^{32}$, soutenu financièrement par le consulat de France et ce de manière stable durant toute la période ${ }^{33}$. L'essor des ordres religieux français et des écoles françaises puise ses sources dans le Protectorat de la France aux chrétiens ${ }^{34}$, «français et catholique sont synonymes» (Riffier 2000: 65). Le Consulat français continue à soutenir les ordres éducatifs destinés à promouvoir la diffusion de la langue. Trois rapports français (de V. Guérin de 1884, de M. Charlot de 1906 et de M. Pernot de 1912) vantent les réussites des établissements français ${ }^{35}$.

Ces écoles sont au coeur d'un « réseau » français dans la ville sainte, autour de la porte $\mathrm{Neuve}^{36}$. Une vie française se dégage autour de la vie de ces établissements, qui s'inscrivent à l'intérieur des remparts mais aussi à l'extérieur. Ils ne sont alors que des "pierres » dans le vaste édifice des oeuvres françaises d'éducation et d'assistance ${ }^{37}$. C'est ainsi que l'on voit s'élaborer, dans les bureaux du Quai d'Orsay, les services davantage liés à la langue et aux établissements à l'étranger. La théorie du patronat de la France sur les latins et les orientaux unis de nationalité ottomane est mise à mal, au moment où le Protectorat est lui-même battu en brèche ${ }^{38}$, et donc la langue française.

Fig. 1 : Orphelines de l'établissement des Sœurs de Sion (ANDS, fin de la période ottomane ; en arrière plan, l'Arc de triomphe)

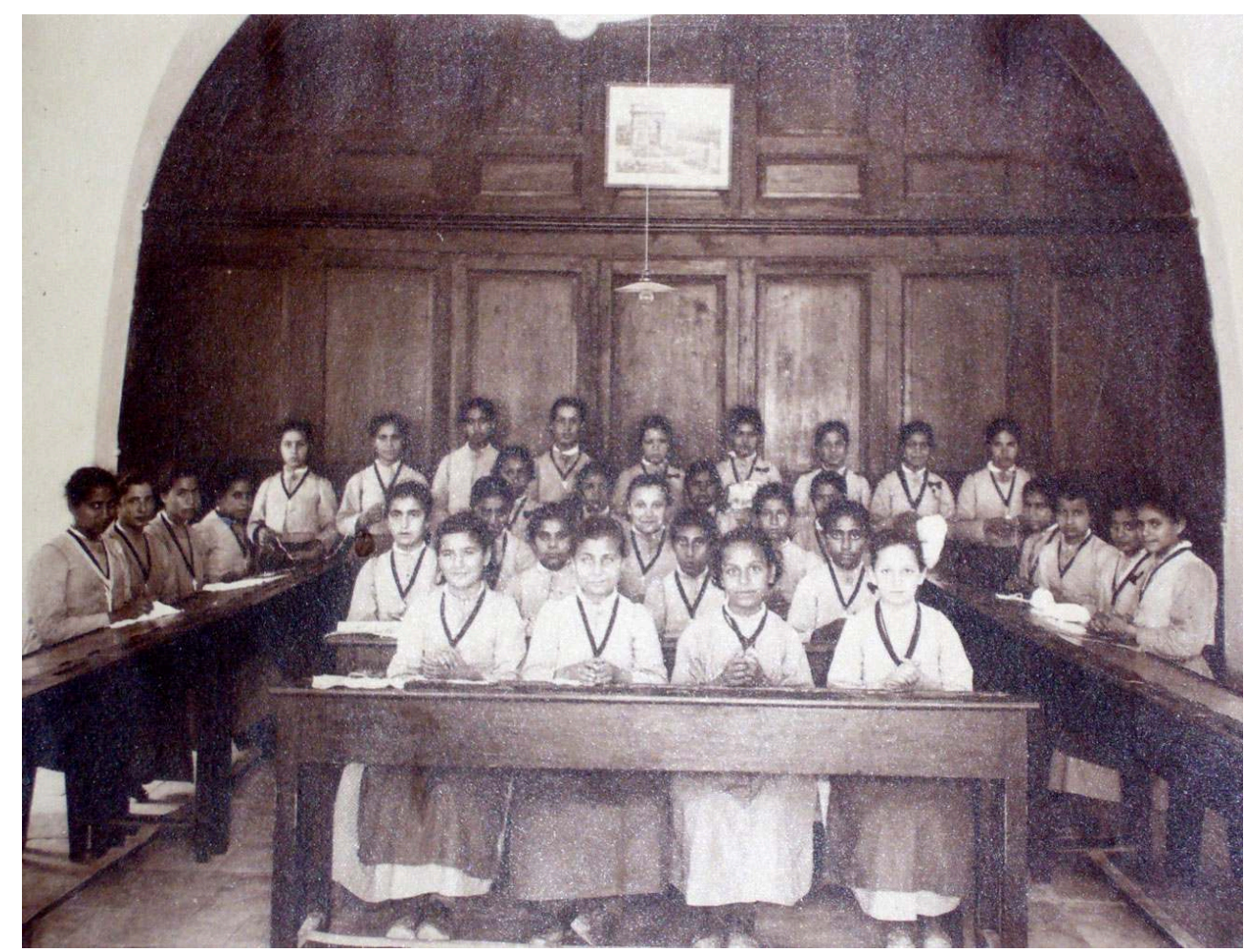


19 On peut avec prudence évoquer la notion « d'aménagement linguistique » dans l'empire ottoman (Thobie 1977) ${ }^{39}$. Ceci contribue en partie à cet essor des écoles étrangères. La France acquiert un rôle important du fait du système de protection accordé par le pouvoir ottoman à la France ${ }^{40}$.

\section{Arabe versus français?}

20 Le curriculum proposé, pour les garçons et pour les filles est multilingue, avec des variations à caractère professionnel ${ }^{41}$. Pour les filles, l'apprentissage du français constitue une valeur sur le marché matrimonial, et reste donc prédominant. Contrairement au système anglais ou grec ${ }^{42}$, le bilinguisme n'est pas mis à l'honneur. Il s'agit d'un apprentissage de langue maternelle française, sans recours à la langue maternelle arabe. Toutes les matières sont en effet enseignées en français. Les Allemands soulignent un risque de déperdition identitaire. "Dans l'exercice des langues, le plus grand intérêt doit, comme il est conforme à la nature, revenir à la langue maternelle [...] les enfants doivent désapprendre à se jeter sur les miettes des langues étrangères [...] Mieux vaut former de bons Arabes que de mauvais Européens [...] des caractères que des carrières [...] du diamant brut que de brillantes imitations» ${ }^{43}$. Pourtant certaines pressions sur les récepteurs de cet enseignement (la Nahda, renaissance autour de la langue arabe, digne d'être parlée et écrite, par exemple) changent progressivement le rapport entre le coût de l'apprentissage et sa valorisation, revalorisant l'arabe.

21 Les établissements français se distinguent par contre pour leur méthode directe et simultanée et une pédagogie différenciée précoce pour l'apprentissage du français et des langues étrangères. 
Fig.2: Relevé de notes d'une élève des Sœurs de Sion, 1904-1905 (ANDS)

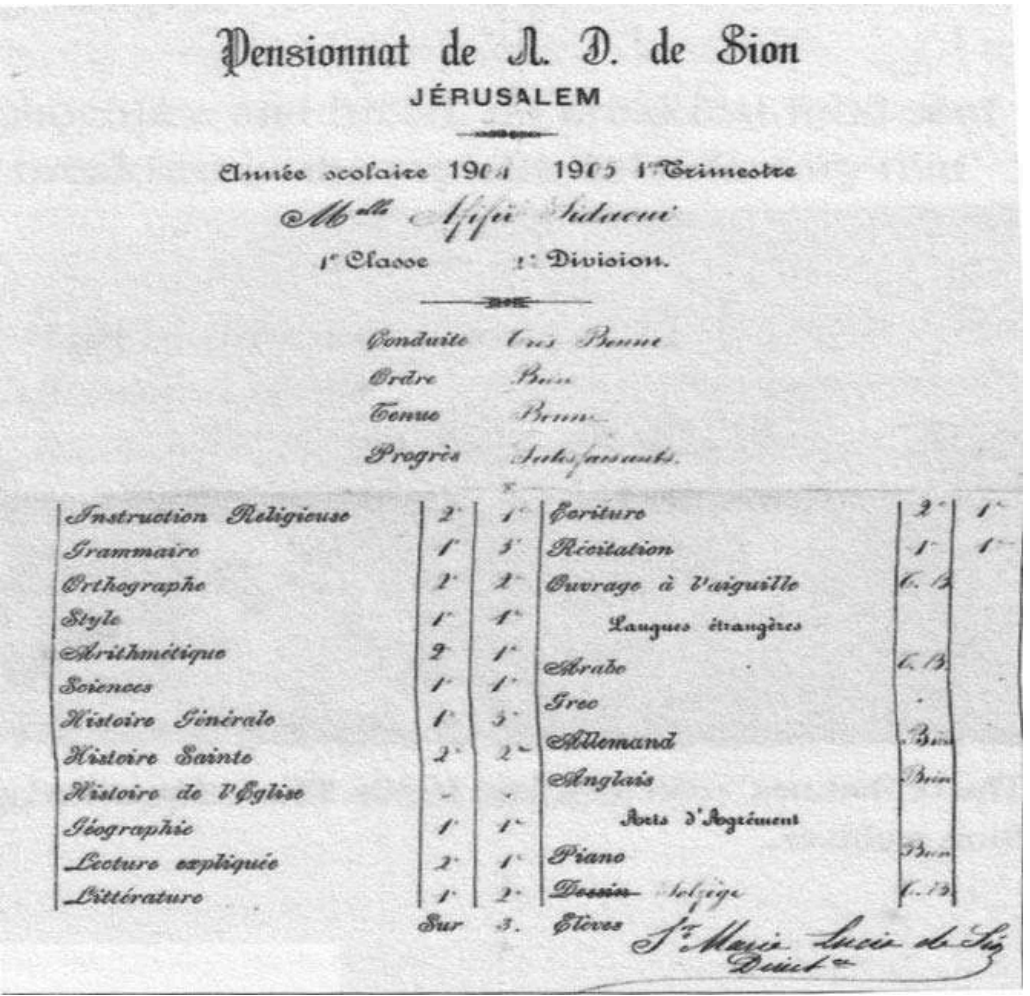

22 La demande des parents se renforce pour un apprentissage de l'arabe. Son apprentissage est renforcé depuis 1904 à l'Ecole normale de Bethléem, où il est enseigné par des instituteurs davantage formés ${ }^{44}$. Elle forme aussi en arabe, de peur de l'internationalisation de l'ordre des Frères, du fait des difficultés éprouvées depuis 1901, pour le recrutement et la formation en métropole. Pourtant le français n'est pas la langue de la religion, il n'y a guère de paroisse francophone pour les fidèles. 
Fig.3 : Ecole normale des Frères, Bethléem (ACB)

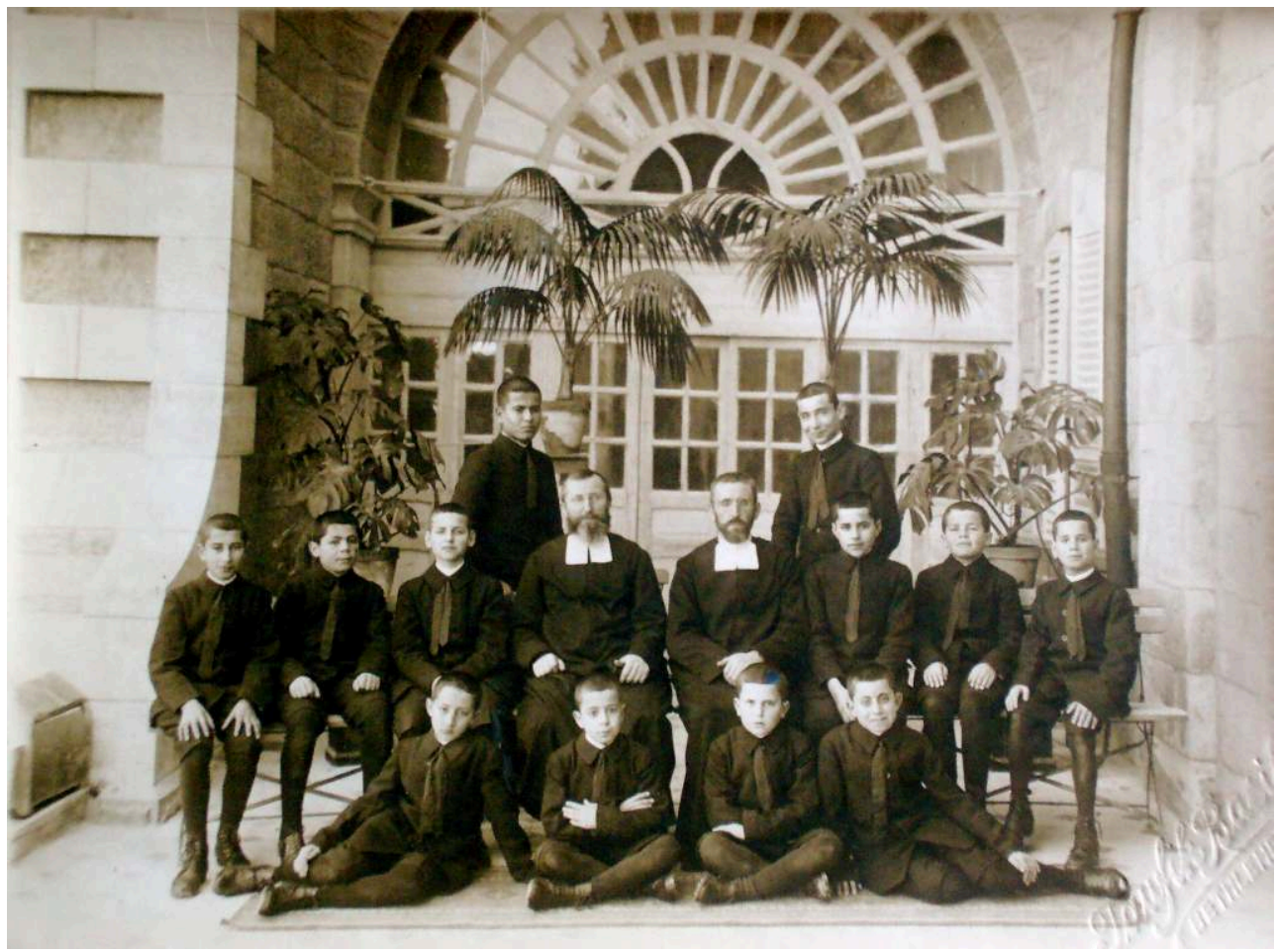

\section{La figure du passeur de langue, passeur de religion?}

Ciments de l'apprentissage et agents de la transmission de la culture française et de la religion catholique, les enseignants missionnaires français n'éprouvent la nécessité d'apprendre activement la langue arabe. Ils ne sont pas formés en arabe avant leur arrivée (ils choisissent d'ailleurs rarement la destination de leur pays de mission). Contrairement aux revendications allemandes, les écoles missionnaires comportent peu de religieux indigènes. Le pourcentage de Frères français au collège de Jérusalem par exemple est révélateur de cet "esprit français » (avant 1904: 90\% de Français; après 1905: entre 80 et $90 \%$; après 1919: entre 45 et 70\%) ${ }^{45}$. Tout comme les Sœurs (les deux ordres précédemment cités comportent un nombre de sœurs " autochtones » un peu plus important) - palestiniennes, syriennes, libanaises) ${ }^{46}$ ils font généralement appel à des professeurs civils, peu formés, pour l'apprentissage de l'arabe (la professionnalisation de cet enseignement et de ce personnel voit le jour surtout sous le Mandat britannique). Pourtant, à la fin de la période ottomane, les ordres missionnaires français sont poussés par le patriarcat à enseigner en arabe, incitation qui se fera plus injonctive dès 1919 et $1920^{47}$.

Le français reste, dans une certaine mesure, une religion " d'évangélisation ", face à ce qui est perçu comme la «menace protestante » et la prépondérance de l'anglais. Dans le cas des écoles pour filles, le caractère religieux est davantage marqué. Pourtant, cette notion appelle à une nuance, il ne s'agit pas d'un prosélytisme ouvert. Il n'est en effet pas à l'ordre du jour pour les élèves musulmans et juifs. La papauté insiste quant à elle sur le respect des chrétiens orientaux ${ }^{48}$. Il s'agit pour les sœurs de «former de bonnes mères et de bonnes chrétiennes ». Les Sœurs de Saint Joseph tentent «d'éviter » que leurs élèves ne tombent dans l'orthodoxie ou le protestantisme par des mariages 
mixtes ${ }^{49}$, mais tout comme les Sœurs de Sion, elles insistent davantage sur les comportements à influencer, les valeurs à transmettre, que sur un prosélytisme actif. «Elles voulaient atteindre l'âme, et pour ce faire elles comprirent que l'unique moyen était l'école» (Jaussen 1927) ${ }^{50}$. Les Sœurs de Saint Joseph s'appuient d'ailleurs davantage que les établissements de garçons sur les exemples religieux au sein des cours de langue (française et arabe) ${ }^{51}$. Les jeunes filles prennent conscience de l'altérité sur les bancs des salles de classe mais aussi à travers les congrégations catholiques, qui sont aussi des lieux de sociabilité religieuse en langue française généralement, telles les Croisées, les jeunes filles de Marie, les Agneaux de Dieu. Les Sœurs de Sion accueillent plus tôt des élèves juives et musulmanes, du fait de leur vocation.

Les établissements des Sœurs accentuent petit à petit leur caractère multicommunautaire au début du siècle ${ }^{52}$. Les établissements pour garçons le sont plus tôt, et les Frères doivent défendre cette vision de l'éducation auprès du Saint-Siège à plusieurs reprises ${ }^{53}$.

\section{Conclusions} fin de la période ottomane (la réouverture est difficile pour les établissements français,
endommagés et nécessitant une aide financière) ${ }^{54}$. On ne peut guère évoquer pour autant la fin du multilinguisme qui a caractérisé la période ottomane, celui-ci s'oriente davantage vers les langues dominantes et bientôt officielles. L'anglais et l'arabe sont au centre des préoccupations linguistiques, même si la langue française n'est pas encore une langue dominée. La période ottomane est annonciatrice des enjeux entre pouvoirs politiques et usages des langues, entre les acteurs du maintien d'une langue ou du support de la diffusion d'une autre. Avant les affrontements de la première guerre mondiale, les tensions sont croissantes entre les deux puissances qui ont de l'influence dans la zone, la France et la Grande-Bretagne ${ }^{55}$, la langue constitue un de ces terrains d'affrontement (de toute évidence porteuse d'identité, elle-même façonnée). La papauté et le patriarcat latin se rangent du côté de la langue arabe ${ }^{56}$.

Reprenant l'image de la valeur monétaire de la langue énoncée par F. Abécassis pour la société égyptienne (Abécassis 2006 : 279- 302), et le terme de «dévaluation » de P. Bourdieu (1982 : 35), on peut également affirmer dans le cas palestinien que dans le marché linéuistique, "inondé » par plusieurs langues, le français a perdu de sa valeur dans l'apprentissage ainsi que sa fonction de lingua franca. Cependant, bénéficiant encore d'une certaine renommée jusqu'au milieu des années 1930, les écoles missionnaires françaises restent les plus importantes (elles scolarisent 2,5 fois plus d'élèves que les écoles anglaises) ${ }^{57}$. Avec l'arrivée des Britanniques la façon de penser les langues entre elles change, l'hébreu et l'arabe deviennent des langues officielles. La loi des autorités mandataires britanniques affecte le statut de la langue française dès 1924 (elle n'est alors plus une langue officielle d'examens) ${ }^{58}$. Le recoupement de plusieurs données sur les rapports des langues entre elles, notamment juridique (le statut du français), fonctionnelle (l'utilité de la langue pour accéder à certains postes), diachronique (la transmission intergénérationnelle), symbolique (le prestige attaché à la langue), et conflictuelle (la domination de la langue sur les autres) (Laponce 2006 : 113) permet d'affirmer qu'à la fin de la période ottomane, le français, alors inséré dans 
un réseau plus ponctuel que global, tend à devenir, à Jérusalem, une langue minoritaire.

Les écoles ont le sentiment de se battre pour la sauvegarde d'un îlot linguistique ; dans les années 1930, certains missionnaires perçoivent la situation comme un "désarroi linguistique $\aleph^{59}$, qui s'est progressivement éloignée sur le terrain, de l'exhortation formulée par un de ces principaux représentants au début du Mandat: «cette langue peut subir une éclipse, ce ne sera jamais une éclipse totale. Et le rayonnement de vie que lui gardent nos écoles françaises, n'est-ce pas la plus comblante des visions en même temps que la plus rassurante des perspectives pour l'avenir? ${ }^{60}$

Fig.4 : Nombre d'habitants de Jérusalem par langue maternelle, 1922 (PRO)

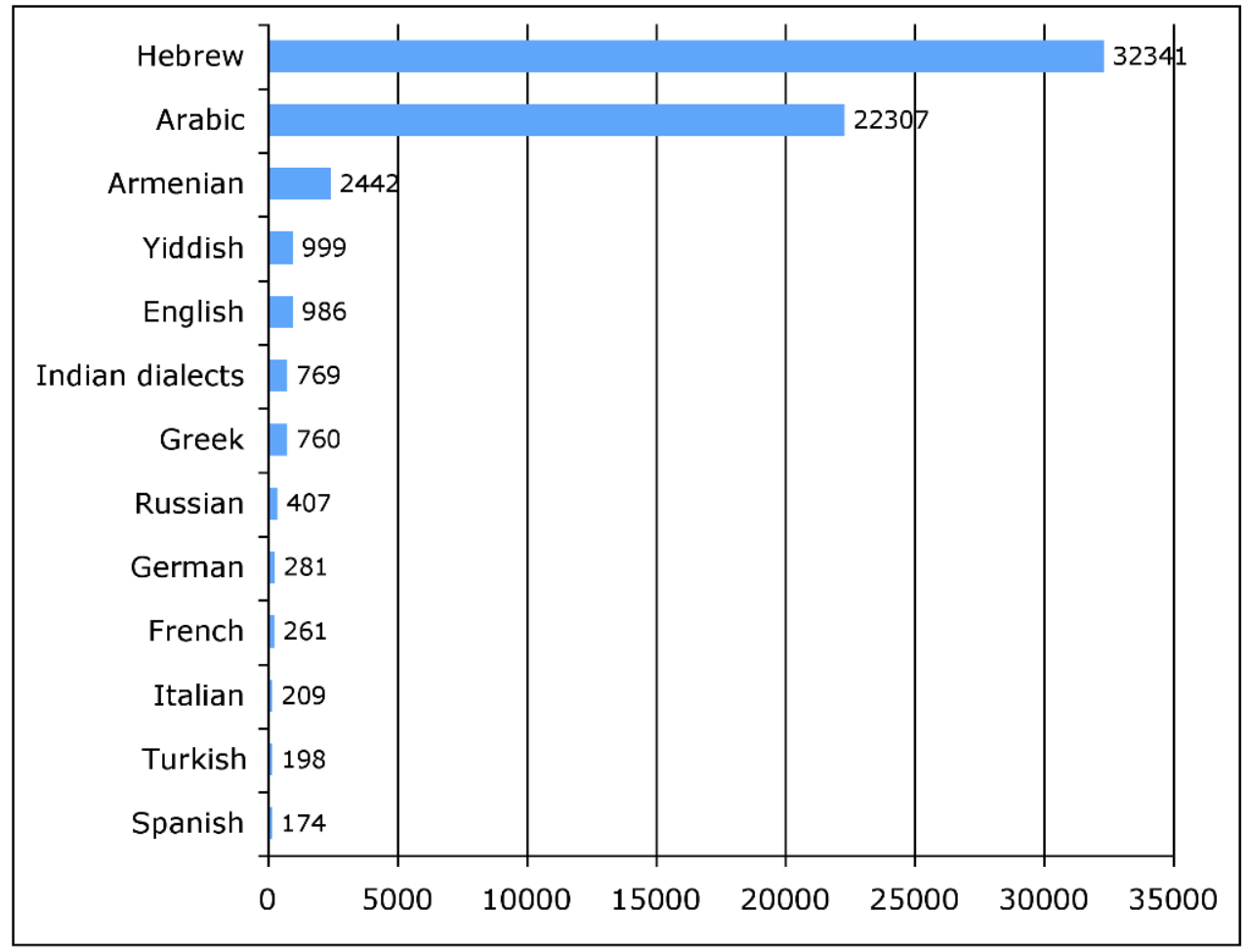

\section{BIBLIOGRAPHIE}

\section{Bibliographie}

ABECASSIS, Frédéric. [2006]. «L'enseignement français en Egypte dans les années 1930 : les dévaluations contraintes d'un modèle ", in P. Cabanel, Une France en méditerranée, Ecoles langues et culture françaises, XIX ${ }^{e}$-XXe siècles. Paris : Creaphis, 279-302. 
ASTAFIEVA, Elena. 2003. « La Russie en Terre sainte : le cas de la société impériale orthodoxe de Palestine (1882-1917)». Cristianismo nella storia 24 (1) : 45.

BEN ARIEH, Yehoshua. 1984. Jerusalem in the XIXth century, the old city. Jerusalem : I. Ben Zvi. BOURDIEU, Pierre. 1982. Ce que parler veut dire. Paris : Fayard.

CUINET, Vital, 1895. La Turquie d'Asie, géographie administrative, statistique, descriptive et raisonnée de chaque province de l'Asie mineure. Paris : E. Leroux.

DHORME, P. 1912. « La langue française en Palestine ». AMAE, Nantes, SOFE, O172, mai 1922.

FORTNA, Benjamin. 2002. Imperial classroom, : Islam, the State and Education in the late Ottoman Empire. Oxford : Oxford University Press.

FRIJHOFF, W. 2008. « La vitalité d'une langue se mesure à sa capacité d'expansion, de concurrence avec les autres langues ", in Geneviève Zarate et al., Précis du plurilinguisme et du pluriculturalisme, Paris : EAC.

GREENBERG, Ela. 2004. « Educating Muslim girls in Mandatory Jerusalem ». Journal of Middle East Studies $36: 1-19$.

KHALIDI, Rashid. 1997. L'identité palestinienne, la construction d'une conscience nationale moderne. Paris : La Fabrique.

JAUSSEN, Antonin. 1927. Coutumes palestiniennes : Naplouse et son district. [ville] : Paul Geuthner.

LANGLOIS, Claude. 2001. «Les congrégations françaises de Terre Sainte au XIXe », in D. Trimbur et R. Aaronsohn, De Bonaparte à Balfour, La France, L'Europe occidentale et la Palestine (1799-1917). Paris : CNRS éditions.

LAPONCE, Bernard, 2006. Loi de Babel et autres régularités de rapports entre langue et politique. SainteFoix : PU Laval.

LAURENS, Henri. 2002. La question de Palestine, tome 1. Paris : Fayard.

-----2004. Orientales III, parcours et situations. Paris : CNRS éditions.

MURRE VAN DER BERG, Heleen. 2006. New Faith in Ancient Lands, Western missions in the Midle East in the XIXth and Early Twentieth centuries. Leiden : Bill, 32 (introduction).

NICAULT, Catherine. 1999. Jérusalem (1850-1948) : des Ottomans aux Anglais, entre coexistence spirituelle et déchirure politique. Paris : Autrement.

PERNOT, Maurice. 1912. Rapport sur un voyage d'étude à Constantinople, en Egypte et en Turquie d'Asie (Janvier- août 1912), Comité de défense des intérêts français au Proche-Orient. Paris : Firmin Didot.

PRUDHOMME, Claude. 2006. « Missions catholiques et rivalités culturelles en Méditerranée orientale (1870-1914) » in P. Cabanel, Une France en méditerranée, Ecoles langues et culture françaises, $X I X^{e}-X X^{e}$ siècles. Paris : Creaphis, 33-54.

RIFFIER, Jean, 2000. Les œuvres françaises en Syrie (1860-1923). Paris : L’Harmattan.

SANBAR, Elias.2003. Figures palestiniennes, une identité en devenir. Paris : Cerf.

TANNOUS, Izzat, 1988. The Palestinians, eyewitness history of Palestine under British mandate. IGT.

THOBIE, Jacques. 1977. « La France a-t-elle une politique culturelle dans l'empire ottoman à la veille de la première guerre mondiale ? » in Intérêt et impérialisme français dans l'empire ottoman 1895-1914. Paris : Publications de la Sorbonne. 
TRIMBUR, Dominique. 2004. Des Européens au Levant- Entre politique, science et religion (XIXe-XXe). Munich : Oldenburg.

\section{NOTES}

1. Abréviations employées par la suite : ACJ (Archives du collège des Frères de Jérusalem), AMAE (Archives du ministère des Affaires étrangères), PRO (Public Record Office), AMG (Archives de la Maison Généralice des Frères des écoles chrétiennes), ANDS (Archives privées de Notre Dame de Sion), APF (Archives de la Propagation de la Foi), ASSJ (Archives privées des Sœurs de Saint Joseph).

2. Ces différents usages ne sont pas perçus comme contradictoires mais comme complémentaires durant la période ottomane.

3. Nous n'évoquerons pas ici les débats qui mettent en scène des pédagogues locaux, tels $\mathrm{K}$. Sakakini, qui fonde l'école nationale Dusturiyya, à partir de 1909. Ces écoles proposent un enseignement en arabe et une focalisation sur la culture arabe et non plus essentiellement européenne. Nous nous intéresserons aux débats entre modèles missionnaires européens par rapport à la langue des missions et à la langue maternelle des élèves.

4. Le pouvoir ottoman va jusqu'à créer, sous l'impulsion directe de Victor Duruy, un lycée d'élite à Istanbul, $c f$. doctorat de Gulsun Guvenli (introduction), sur le lycée de Galatasaray d'Istanbul, soutenu en février 2008 à l'EHESS (non publiée). Les Jeunes Turcs auraient eu l'intention de confier aux Frères français des écoles chrétiennes les Ecoles normales du Liban, lettre du Frère Onésime à la Propaganda Fide, 1910, AMG, NH 701. Par certains aspects, le français semble donc participer en partie au processus « d'ottomanisation » défini à Istanbul.

5. Le consulat britannique ouvre ses portes en 1838, le consulat de France en 1843, et devient Consulat Général en 1891 avec davantage de pouvoirs puisque dépendant directement du MAE. "Jérusalem est depuis longtemps la ville des églises et des couvents; on pourrait présentement l'appeler la ville des écoles car ardent est le zèle pour la formation de la jeunesse qui s'est réveillé pour ne pas dire enflammé chez toutes les nations et les confessions. Bientôt la ville ne sera qu'une immense université. » ACJ, Institut archéologique allemand, 1905 (extrait de l'annuaire de la Palestine de l'Institut évangélique allemand d'archéologie de Terre Sainte à Jérusalem; les écoles populaires arabes de Jérusalem, par G. Eberhard, recteur à Zarrentin, Meclembourg).

6. C'est donc la ville de Palestine où les luttes d'influence entre les grandes puissances sont les plus fortes. L'expansion et la modernisation du système éducatif sont parallèles à celles du gouvernement et de l'armée, mais les demandes sont plus nombreuses que les places disponibles. Liant charges administratives et religieuses des notables, ce statut fait de la ville la capitale de la Palestine avant même qu'elle ne devienne une entité administrative et politique séparée en 1918. Elle acquiert le statut de sandjak (capitale de district) autonome en 1872. Elle est un centre de gouvernement, d'étude et consulaire, ainsi que du tourisme, des pèlerinages mais son commerce et son industrie ont une croissance moins forte que celle des villes littorales (Khalidi $1997: 86$, et Sanbar 2003 : 193).

7. L'expression est de P. Cabanel. Dès 1856 (fin de la guerre de Crimée, traité de Paix de Paris), la Sublime Porte accorde des terrains à la France et facilite l'installation des communautés religieuses françaises (en 1901: accords de Mytilène, 1913 : accords de Constantinoplepréservation des droits des communautéschrétiennes). Toutes ces activités apportent un équilibre à la ville par rapport à l'essor du littoral palestinien (Laurens 2002 : I, 75-77. Jérusalem est donc à la marge de l'Europe occidentale, mais par un jeu de miroir, lui est étroitement lié, et représente bien plus que de simples intérêts linguistiques pour les nations concernées. 
8. Statistiques fournies à la Maison Généralice en 1905, 1912 et 1921, AMG, NH 701, nº13 et ACJ, ASSJ, ANDS. Ces chiffres sont similaires à ceux envoyés au Consulat Général de France à Jérusalem. Le détail des différents ordres éducatif est fourni dans la deuxième partie de l'exposé. 9. Pour la période ottomane on peut aller jusqu'à évoquer une « francisation ».

10. Les élites qui appartiennent à l'administration ottomane venues de Palestine semblent davantage attachées aux écoles religieuses musulmanes, avant la première guerre mondiale, David Kushner. 1988. Palestine in the late Ottoman period : political, social, economical transformations. Leiden: Brill, p. 152. Après la première guerre mondiale, les élèves musulmans sont plus nombreux au sein des écoles françaises pour garçons ainsi que dans les établissements pour filles. 11. Cette expansion est également soutenue par l'Oeuvre des Ecoles d'Orient, créée en 1851.

12. Sont évoqués alors dans certains rapports, tels le rapport Charlot de 1906 et le rapport Pernot de 1912, le « prestige séculaire de la France au Levant, le protectorat exercé [...] sur les Chrétiens de l'empire ottoman, la prépondérance des représentants de la France dans les affaires de la Sublime Porte et dans la défense des minorités, et surtout l'immense rayonnement de la bienfaisance française, par les hôpitaux, les écoles, les oeuvres de toutes les sortes ", autant de causes qui ont créé en Palestine, d'après les auteurs de ces rapports, ce besoin du français.

13. Le P. Dhorme se fait l'écho de l'idée selon laquelle la maîtrise du français est consécutive à la classe sociale, «c'était le signe qu'on était sorti du niveau moyen », AMAE, Nantes, SOFE, O172, « La langue française en Palestine », 18 mai 1922.

14. 22000 habitants en 1870, 31000 en 1880, 50000 en 1900, enfin 70000 en 1914, s'accompagnant à partir de 1892 de modernisations de ses structures urbaines (Sanbar $2003: 43$ ). L'anglais apparaît déjà à la fin du XIX" ${ }^{\mathrm{e}}$, comme une langue «fédératrice " (Tannous 1988 : IGT). L'auteur évoque la création de l'école Saint George's à la fin du XIX et l'importance de la culture sportive qui lui est liée.

15. Le lycée impérial ottoman n'ouvre ses portes que durant la première guerre mondiale, dans les locaux du collège des Frères, et ferme après la défaite ottomane dès 1 afin de 1917, ACJ, Historique,1880-1900 ; Cuinet 1894-1895 et AMAE, série A, 78. Le Wali entretient des liens très cordiaux avec l'ordre français des Soeurs de Saint Joseph et d'autres ordres religieux français, ANDS, Jérusalem, Annales, 1872.

16. Ces établissements comportent généralement deux sections: une section payante plus hétérogène et une section gratuite qui continue à accueillir une majorité de catholiques plus pauvres.

17. ACJ, Institut archéologique allemand, 1905.

18. ACJ, Institut archéologique allemand, 1905. Mais si la France est présente au niveau économique dans la ville et, à une plus grande échelle dans la région de Jérusalem et Jaffa, notamment dans les secteurs bancaires (la branche du Crédit lyonnais ouvre en 1863), les concessions des premières lignes de chemin de fer (la ligne de chemin de fer Jérusalem- Jaffa est ouverte en 1892), son importance est toute relative pour de nombreux observateurs. «Allemands, Italiens et Autrichiens trouvent le moyen d'y faire d'excellentes affaires, les nôtres n'en font pas on est porté à conclure que la France produit peu et qu'elle a peu de commerce. Les produits français ne se trouvent nulle part, l'hôpital français achète toute sa pharmacie à des voyageurs allemands » (Pernot $1912: 131)$.

19. Depuis 1862 , beaucoup d'élèves juifs sont scolarisés en français dans les écoles de l'AIU (Alliance Israélite Universelle).

20. D'autres collèges existent, dont celui du Bishop Gobat fondé dès 1847, celle de Saint George's, fondée en 1899; $c f$ le tableau de recension des établissements, d'après les données fournies par l'Institut archéologique allemand en 1905, ACJ (Tab.2). L'école ottomane islamique est créée en 1906 (Fortna 2002).

21. La Turquie d'Asie, géographie administrative, statistique, descriptive et raisonnée de chaque province de l'Asie mineure, Paris : E. Leroux. A l'époque ottomane deux ordres surtout assurent 
70\% de l'enseignement : il s'agit des Sœurs de Saint Joseph et des Sœurs de Sion. Dès 1890 elles sont présentes sur l'ensemble du territoire palestinien. L'action éducative de ces ordres féminins s'accompagne généralement d'une action sanitaire auprès des populations urbaines mais aussi rurales. Les écoles musulmanes sont encore peu nombreuses jusqu'au début du XXe siècle, la première école ouvre ses portes en 1895, et accueille un petit nombre d'élèves (Greenberg 2004 : 1-19). En 1895, V. Cuinet ne recense que 100 filles scolarisées à Jérusalem et 50 à Jaffa.

22. Peu de listes complètes d'élèves ont été conservées pour l'époque ottomane.

23. Nous ne considérerons pas les raisons et les modalités politiques de la mise en place des différents systèmes éducatifs, dont l'ensemble des écoles catholiques françaises, qui sortent du cadre de notre étude, mais nous nous y référerons.

24. Le consul de France condamne, dès l'ouverture des établissements britanniques, la rivalité ouverte de l'Angleterre qui, par le biais de ses écoles, fait « mille efforts, impuissants, il est vrai, jusqu'à ce moment, pour créer en Palestine, par le prosélytisme, une nation anglaise. » Il note la qualité de leur système éducatif, qui a pour lui « une supériorité incontestable sur tous les autres chrétiens indigènes ", APF, 0082, E20, Le Consul de France à Jérusalem à la Propagation de la Foi, 16 mars 1845, il y critique vivement l'éducation dispensée par les franciscains.

25. Pendant la période ottomane, les langues dominantes au sein de la population de Jérusalem ne sont pas officielles dans l'empire ottoman, ce qui implique un rapport difficile à la langue turque que beaucoup de Palestiniens, même issus des couches les plus aisées de la population, ne maîtrisent guère. Cependant, ce statut des langues minoritaires implique que des libertés politiques et linguistiques sont reconnues aux différentes communautés par l'administration ottomane.

26. «Les écoles françaises, presque toutes entre les mains des ordres religieux d'hommes et de femmes étaient sans cesse obligées de s'étendre ou de se multiplier pour satisfaire à ce besoin. Les établissements rivaux quelle que fût leur origine, devaient faire entrer le français dans leurs programmes. Les oeuvres indigènes faisaient passer le français aussitôt après l'arabe dans leurs préoccupations scolaires». AMAE, Nantes, SOFE, 0172, P. Dhorme, «La langue française en Palestine ", 18 mai 1922.

27. En 1900, 11 écoles gouvernementales pour garçons (soit 3761 élèves), 38 écoles privées chrétiennes (soit 6630 élèves) et 7 écoles privées musulmanes (soit 1101 élèves) (Riffier 2000 : 281). Ce système est contrôlé par le ministère de l'éducation à Istanbul, tandis que les écoles privées musulmanes, juives et chrétiennes, préalablement approuvées par ce ministère, sont plus autonomes. Il est officiellement ouvert aux élèves de l'empire de toutes religions et de tous groupes ethniques, mais dans les faits si l'on considère les chiffres de plus près, la plupart des élèves sont musulmans. C'est la langue turque qui est fondamentale dans cet apprentissage. De nombreux élèves sont tentés d'effectuer leur scolarité dans les établissements missionnaires, où l'apprentissage du turc est facultatif (même remarque pour l'enseignement des filles). La réglementation pour l'Instruction Publique est promulguée le 1er septembre 1869.

28. L'expression est du linguiste L.J. Calvet.

29. ACJ, rapport de l'institut archéologique allemand, 1905.

30. Le fait que les élèves intègrent tout type de position dans l'administration et qu'il leur soit possible d'accéder à la plupart des postes indique leur niveau, moins spécialisé que celui des élites libanaises.

31. Handbook of Palestine, 1927, p 179

32. 1848 Soeurs de Saint Joseph (Jérusalem, 1849 Jaffa, 1853 Bethléem, 1872 Ramleh, 1873 Ramallah, 1875 Beit Jalah, 1889 Nazareth, 1904 Naplouse) ; 1855 Dames de Nazareth ; 1856 Soeurs de Sion (1874 Saint Pierre de Sion pour garçons) ; 1876 Frères des écoles chrétiennes (Jérusalem, 1882 Jaffa, 1884 Caiffa, 1893 Bethléem et Nazareth) ; 1880 Soeurs du Rosaire ; 1886 Filles de la Charité (Saint Vincent de Paul) ; 1909 Franciscaines missionnaires de Marie. 
33. Même en période de tensions aigues entre les missions catholiques et le gouvernement de la IIIe République, des honneurs sont rendus aux ordres religieux en Palestine. C'est notamment le cas pour le fondateur des collèges des Frères de Palestine, le Frère Evagre, qui obtient entre autres les Palmes d'officier d'académie, et le Brevet d'officier de l'Instruction Publique (le P. Dhorme est décoré de la légion d'honneur).

34. Dans les accords internationaux, le Protectorat catholique français est un privilège reconnu à la France en 1740, de protéger partout, notamment dans les lieux saints, les personnes et les oeuvres catholiques latines étrangères, et quelle qu'en soit la nationalité, AMAE, Nantes, série A, 78 THOBIE, Jacques, 1977. « La France a-t-elle une politique culturelle dans l'empire ottoman à la veille de la première guerre mondiale?» in Intérêt et impérialisme français dans l'empire ottoman 1895-1914, Paris : Publications de la Sorbonne, p 366. En 1901, pour obliger le sultan à honorer certaines dettes et à accepter les exigences de sociétés françaises, Paris monte une intervention navale qui aboutit à l'occupation des douanes de l'île de Metelin. Ce coup est l'occasion, pour le gouvernement français, d'exiger de Constantinople la délivrance de firmans à tous les établissements scolaires, hospitaliers et religieux agrandis ou construits sans autorisation depuis le traité de Berlin (1878). En 1913, dans le cadre des négociations qui mènent au grand emprunt de consolidation et qui définissent les zones d'influence étrangère dans l'Empire, Paris impose l'extension ou l'acquisition de plusieurs privilèges pour tous les établissements français ou protégés dont la liste est soigneusement établie (exemption des taxes municipales directes, possibilité pour les établissements reconnus de posséder tous les immeubles -affranchis de l'impôt foncier). Cet accord établit des conditions très favorables à une accélération de la progression de l'enseignement de la langue, de l'histoire et de la culture française dans l'empire ottoman.

35. M. Charlot est envoyé par une commission française dont le but est la diffusion de la langue française; s'il critique de manière globale les méthodes d'apprentissage des établissements congréganistes, il insiste sur l'influence et aussi l'efficacité de ces établissements auprès de la population de la vieille ville. Le rapport Pernot, élaboré dès 1912, à la suite du voyage du délégué du Comité pour les intérêts français, vante l'enseignement des Frères et des Sœurs sur plusieurs points, et en particulier le prestige, le nombre d'élèves et les diplômes.

36. La porte Neuve est ouverte en 1889, à l'initiative de la France. Cette ouverture est significative de l'importance de la présence «spatiale » de la France.

37. Rapports au MAE, et Riffier 2000.

38. Le traité de Berlin (1878), tout en réservant les droits de la France, avait reconnu à chaque puissance le droit d'exercer partout, au profit de ses propres nationaux, ce type de protection.

39. L'auteur évoque davantage la naissance d'une "politique culturelle» dont l'aspect linguistique constitue une branche.

40. Capitulations: exemptions juridictionnelles et fiscales s'appliquant aux ressortissants «francs » des Echelles du Levant, muées au XIXe pour des individus ou groupes communautaires, statut d'extraterritorialité, garanti par consuls européens.

41. Selon les Annales des Soeurs de Saint Joseph et des Frères des écoles chrétiennes, curriculum commun (écoles payantes):

Français (lecture, dictée, grammaire, composition, morale), arabe, anglais

Histoire, géographie, dessin, musique, gymnastique

Mathématiques (arithmétique, algèbre, géométrie)

Pour les filles: couture, broderie, tricot (autres langues étrangères: allemand et grec) ; pour les garçons: sciences (physique, chimie, sciences naturelles), dactylographie, comptabilité.

42. Au sein des écoles catholiques, différence notable avec les écoles paroissiales tenues par les franciscains (et plus tard le Terra Sancta college qui accueille les élites sous les Mandat) : l'arabe est mis à l'honneur, Fra d'Aleppo élabore des ouvrages en arabe, ACJ, Institut archéologique allemand, 1905. 
43. ACJ, Institut archéologique allemand, 1905.

44. ACB, dossier « Ecole normale ».

45. $\mathrm{ACJ}$ et $\mathrm{AMG}$, Etats jaunes : relevés statistiques du personnel.

46. Il est intéressant d'observer dans ce contexte linguistique la naissance de l'ordre éducatif local des Sœurs du Rosaire, issu des Sœurs de Saint Joseph, animé par le chanoine du Patriarcat latin Don Tannous. Les Sœurs enseignent en arabe essentiellement et consacrent quelques heures à langue française, davantage enseignée comme une langue étrangère. En 1895, plus de $70 \%$ d'entre elles sont françaises dans les sections payantes ( $50 \%$ en moyenne dans les sections gratuites), cette proportion ne change guère durant la période considérée, Cuinet, Vital, op.cit., $p$ 526.

47. Encyclique Maximum Illud de 1919 et AMG, Patriarcat latin de Jérusalem, note aux écoles catholiques sur le catéchisme.

48. Encyclique de 1894, Dignitas Orientalum, de Léon XIII. Les Sœurs de Saint Joseph s'établissent en dernier dans la ville de Naplouse, elles prennent conscience très rapidement de la difficulté à convertir. ASSJ, Historique de Naplouse, elles désirent notamment répondre à la présence des religieuses anglicanes et protestantes dès 1880 .

49. Les Sœurs de Sion ont pour "vocation première " la conversion d'Israël, mais cette volonté de conversion s'exprime essentiellement par des prières et actions de grâces et non par un prosélytisme actif. De 1883 à 1930, on relève en effet une cinquantaine d'abjurations d'élèves orthodoxes et protestantes et une cinquantaine de conversions d'élèves juives (dans ce cas, il faut tenir compte de la conversion de certaines familles de 4 à 8 membres, ce qui relativise le nombre total de conversions). Les Annales insistent sur le fait que le légat met plusieurs obstacles avant d'autoriser ces conversions, ANDS, Journal de l'Ecce Homo, et cahiers des conversions et des abjurations (1865-1944).

50. Ceci a également sans doute pour but de rassurer l'opinion publique et les organismes français qui soutiennent financièrement les établissements, de mettre en valeur leur rôle missionnaire.

51. Plusieurs cas apparaissent dans les archives (par exemple une élève protestante anglophone venue pour l'apprentissage du français chez les Sœurs est «touchée » par la méthode, le « souvenez-vous à Joseph » et se convertit au catholicisme, selon ASSJ).

52. Chez les Sœurs de Saint Joseph, les $3 / 4$ des élèves restent catholiques (romaines et orientales de tous rites). Ce recrutement multicommunautaire se maintient, malgré les dangers de placer les filles «chez les Européens» comme le font ressortir les avertissements de certaines organisations musulmanes et juives aux parents. Selon ces organisations, les fillettes seraient plus exposées à "se perdre ", à " lire de mauvais ouvrages " et à des risques de perte de leurs repères identitaires et linguistiques (ANDS, Journal de l'Ecce Homo).

53. AMG, correspondance avec la Propaganda Fide, 1890, 1898, 1910, au sujets des "dissidents » dans leurs écoles.

54. AMAE, Nantes, OBIP, dossier des écoles missionnaires françaises.

55. Le Levant indique ici la zone s'étendant de l'Egypte au nord du Liban (terme utiliser jusqu'à la première guerre mondiale). En 1919, G. Clémenceau, président du Conseil, au début de la conférence de paix, renonce explicitement à la Palestine, mais pas au Protectorat. En décembre 1917, lorsque le général Allenby est escorté par le Haut-Commissaire français François Georges Picot lors de son entrée à Jérusalem, le Protectorat a déjà cessé depuis 1914, date à laquelle les Capitulations ont été abolies. Le traité de San Remo (24 avril 1920) accorde le Mandat français sur la Syrie et le Liban, la France renonce officiellement au protectorat, AMAE, E, Levant, Palestine 8, $\mathrm{n}^{\circ}$ 37, Doulcet à Millerand; AMAE, Nantes, série A, 52 à 57, 78. Mais si Paris se désengage au niveau du Protectorat, elle ne renonce pas à ses prolongements scolaires. 
56. Le nouveau pape Benoît XV à une position très tranchée vis-à-vis du nationalisme des missionnaires et de l'utilisation de la langue nationale au sein des écoles, qualifiant de «peste la plus affreuse » la considération des intérêts des pays des missionnaires.

57. PRO, Annual Report, Education, 1930., « Foreign missions schools ».

58. A partir de 1914, la population juive est 3 fois plus nombreuse que la population chrétienne (45 000, 16000 chrétiens, 11000 musulmans). A partir des années 1920, le mouvement juif nationaliste (notamment $\mathrm{Z}$. Jabotinsky) lance un appel à la langue française, en réaction contre les Britanniques.

59. Dans l'espace public, le français passe du statut de «vitrine de la France » au français langue étrangère, en raison d'une diffusion inopérante.

60. AMAE, Nantes, SOFE, O172, P. Dhorme, « La langue française en Palestine », 18 mai 1922.

\section{RÉSUMÉS}

L'article analyse le multilinguisme, réputé, des écoles de Jérusalem durant le dernier quart du XIXe et au début du XXe siècle dans les écoles missionnaires françaises. Devenant progressivement la capitale politique et administrative de la Palestine à la fin de la période ottomane, Jérusalem est le théâtre, depuis le milieu du XIXe siècle, des ambitions des puissances européennes et des rivalités des missions chrétiennes, perceptibles notamment à travers leur offre éducative. Au sein des écoles, les liens entre les langues reflètent la diversité des élèves, de leurs communautés et celle des enseignants, "passeurs de langue », ainsi que les usages du français dans la société hiérosolomytaine. Il constitue une des clés de la promotion sociale à Jérusalem, produit d'un aménagement linguistique, mais il est progressivement évincé de l'espace public et scolaire. Ces rivalités linguistiques sont également perceptibles à travers la complexité des programmes scolaires, qui doivent faire face à une « inflation linguistique ».

This article analyses the multilingualism of Jerusalem schools during the last quarter of the nineteenth and the early twentieth centuries. Gradually becoming the political and administrative capital of Palestine at the end of the Ottoman period, Jerusalem is the stage, since the middle of the nineteenth century, for a confrontation between European powers and Christian missions rivalries, especially noticeable through the educative offer they propose. Within the schools, the links between languages shed light on the diversity of students, their communities, their teachers and the use of French in the Jerusalem society. French constitutes a key to social advancement; resulting of a linguistic policy, but it is progressively supplanted from the public and the educational spheres. These linguistic rivalries are also visible through the complexity of the curriculum, facing a "linguistic inflation".

\section{INDEX}

Keywords : Christian missions, Jerusalem, multilingualism, rivalry, XIXth century, XXth century

Mots-clés : écoles missionnaires, Jérusalem, multilinguisme, politique linguistique, rivalité linguistique, XIXe siècle, XXe siècle 
AUTEUR

KARÈNE SANCHEZ-SUMMERER

Universiteit Leiden 